\title{
POLARIZABILIDADES E PRIMEIRA HIPERPOLARIZABILIDADES ELÉTRICAS DIPOLARES DE ÉSTERES E-4-AMINO-TRANS-1,3-BUTADIENIL-(1',2'-DI-HIDROXIBENZENO) DE BORO, ALUMÍNIO E GÁLIO
}

\author{
Márcia Barsottelli Procópio e Amary Cesar* \\ Departamento de Química, Instituto de Ciências Exatas, Universidade Federal de Minas Gerais, Av. Antonio Carlos, 6627, \\ 31270-901 Belo Horizonte - MG, Brasil
}

Recebido em 7/5/08; aceito em 12/1/09; publicado na web em 28/5/09

\begin{abstract}
DIPOLE POLARIZABILITIES AND FIRST HYPERPOLARIZABILITIES OF E-4-AMINE-TRANS-1,3-BUTADIENYL-(1',2'DIHYDROXIBENZENE) ESTERS OF BORON, ALUMINUM AND GALLIUM. Static electric dipole polarizabilities and first hyperpolarizabilites have been calculated for the title molecules and their $3^{\prime}$ and $4^{\prime}$-nitro derivatives at ab-initio Hartree- Fock/6$31 \mathrm{G}(d, p)$ level. The influence of the pivotal $p$ vacant $3 \mathrm{~A}$ elements $(\mathrm{B}, \mathrm{Al}$ or $\mathrm{Ga}$ ) substitution on the electrical properties of these molecules is detailed. The axial vector components of the first hyperpolarizabilities $\beta(0)$ of the push-pull $4^{\prime}$-nitro derivatives, $-18.2 \times 10^{-32}$ esu (B), $-21.1 \times 10^{-32}$ esu (Al) and $-20.8 \times 10^{-32} \mathrm{esu}(\mathrm{Ga})$ are calculated to be as much as fourfold larger then that calculated for the $\boldsymbol{p}$-nitroaniline, a reference organic molecule for comparison for this type of molecular property.
\end{abstract}

Keywords: dipole (hyper)polarizabilities; boronates, aluminates and galiates; Hartree-Fock calculations.

\section{INTRODUÇÃO}

Moléculas orgânicas insaturadas contendo um sistema de elétrons conjugados, localizados em unidades individuais ou estendidas na forma de cadeias poliméricas, formam uma grande classe de materiais que apresentam ótimas características óticas não-lineares. ${ }^{1-4}$ A combinação desses sistemas orgânicos com centros metálicos adequados produz, complementarmente, importantes exemplos de derivados organometálicos que desenvolvem valores promissores para os parâmetros que descrevem essas propriedades óticas. ${ }^{5}$

As respostas óticas não-lineares de compostos moleculares originam-se, microscopicamente, nas excitações virtuais que seus elétrons podem sofrer ao se moverem de orbitais ocupados para orbitais não ocupados, em especial, entre os orbitais de valência de menores energias HOMO e LUMO. A facilidade com que essas excitações ocorrem está diretamente relacionada com a capacidade da radiação eletromagnética em interagir com um sistema molecular e, assim, induzir consideráveis transferências de cargas entre o estado eletrônico fundamental e os estados eletrônicos excitados de baixa energia. Essa polarização de cargas favorece o surgimento de polarizabilidades $\alpha(0)$ e hiperpolarizabilidades elétricas $(\beta(0)$ (primeira), $\gamma(0)$ (segunda) etc.) apropriadas para o desenvolvimento de materiais com propriedades óticas não lineares. ${ }^{1,2} \mathrm{~A}$ união de grupos doadores (D) e retiradores (R) eficientes de elétrons, intercalados por um sistema de elétrons $\pi$-conjugados como, por exemplo, o grupo aromático fenila ou a cadeia aberta do trans-1,3-butadienila, formam um conjunto de moléculas (chamados sistemas eletrônicos do tipo push-pull D-R) que apresentam excelentes hiperpolarizabilidades elétricas moleculares. A escolha da combinação adequada do par D e R e a presença de um intercalador insaturado, que atua como caminho para deslocalização dos elétrons é fundamental na construção de moléculas que apresentam maiores parâmetros óticos não-lineares. Usualmente, os grupos amino $-\mathrm{NH}_{2}$, e nitro $-\mathrm{NO}_{2}$ são os mais utilizados como unidades doadoras e retiradoras de elétrons e a $p$-nitroanilina ou o 4-nitro, $4^{\prime}$-aminoestilbeno são exemplos de sistemas de referência com hiperpolarizabilidades moleculares médias. ${ }^{2}$

*e-mail: yrra@ufmg.br
Estudos experimentais e computacionais mostram que os derivados organoboranos contendo um ou dois grupos mesitila (mesitila=2,4,6-trimetilfenila) ligados diretamente a um átomo trivalente de boro, apresentam valores relativamente atrativos para a primeira e segunda hiperpolarizabilidades elétricas. ${ }^{6}$ Nesses compostos, o fragmento borodimesitila atua como um grupo retirador de elétrons, com uma eficiência igual ou maior que o grupo nitro, e com a vantagem extra de poder ser quimicamente modificado por substituições adequadas nas posições 3 e/ou 5 em cada um dos anéis aromáticos. Resultados análogos foram também obtidos para os compostos boroxínicos octupolares em experimentos de geração e espalhamento de harmônicos de luz laser. ${ }^{7}$ A presença de um orbital vacante $p$ propicia ao átomo de boro, inserido em uma cadeia carbônica insaturada, uma participação ativa na dinâmica do sistema conjugado de elétrons, estendendo-lhe a conjugação e permitindo o surgimento de uma pequena ou grande separação de cargas devido ao seu caráter receptor de elétrons. Espera-se que essa característica ímpar dote os compostos organoboranos trivalentes de boas ou ótimas propriedades óticas não-lineares. De fato, Reyes et $a l .^{8}$ estudaram a eficiência da geração de segundo harmônico (SHG) por amostras sólidas (pó) de boranos tetravalentes imino fenólicos do ácido salicílico, e, nesses experimentos, não foram observados sinais SHG para moléculas contendo grupos doadores de elétrons fortes como dietilamina. Somente os derivados metoxi exibiram uma modesta eficiência na geração de um segundo harmônico (SHG).

Paralelamente a essas características óticas de compostos organoboranos moleculares, os materiais boratos inorgânicos distingem-se por apresentarem excelentes respostas óticas não-lineares e são, portanto, sistemas de grande interesse científico e tecnológico. ${ }^{9} \mathrm{Bo}-$ ratos como o pentaborato de potássio tetra-hidratado, $\mathrm{KB}_{5} \mathrm{O}_{8} \cdot 4 \mathrm{H}_{2} \mathrm{O}$, o $\alpha$-diborato de bário, $\alpha-\mathrm{BaB}_{2} \mathrm{O}_{4}$ e o hexaborato de césio e lítio, $\mathrm{CsLiB}_{6} \mathrm{O}_{10}$, encontram aplicações tecnológicas como materiais úteis na manipulação de luz por processos não-lineares. Boratos são, em geral, superiores a outros materiais empregados na modificação das propriedades da luz, como o niobato de lítio, $\mathrm{LiNbO}_{3}$, e o bifosfato de potássio (KDP), $\mathrm{KH}_{2} \mathrm{PO}_{4}$, por apresentarem uma faixa mais ampla de transparência na região do ultravioleta próximo, uma boa estabilidade térmica e química etc. ${ }^{9-11}$ 
Estas perspectivas abrem-nos a possibilidade de propormos derivados organoboratos protótipos não centro-simétricos que possam servir de base para o desenho de moléculas apresentando hiperpolarizabilidades competitivas. Três classes de organoboratos simples foram estudadas anteriormente por nós: os derivados do acido borônico $\mathrm{R}-\mathrm{B}(\mathrm{OH})_{2}$, ésteres boronatos alicíclicos simples $p$-X-fenil(1,3propanodiol)boronato $\left(\mathrm{X}=\mathrm{H}, \mathrm{NH}_{2}, \mathrm{NO}_{2}\right)$ e ésteres aromáticos do tipo $\mathrm{D}-\mathrm{R}$, entre eles, os ésteres $p$-X-fenil[( $\left.4^{\prime}-\mathrm{Y}\right\}-1^{\prime}, 2^{\prime}$-di-hidroxibenzeno] boronatos, $(\mathrm{X}, \mathrm{Y})=(\mathrm{H}, \mathrm{H}),\left(\mathrm{NH}_{2}, \mathrm{NO}_{2}\right),\left(\mathrm{NO}_{2}, \mathrm{NH}_{2}\right),{ }^{12}\left(\left(1^{\prime}, 2^{\prime}\right.\right.$-di-hidroxibenzeno)boronato é também anotado como $1^{\prime}, 2^{\prime}$-benzodioxaborol ou simplesmente catecol boronato). Os resultados mostram que, dentre esses compostos, os ésteres $p$-nitrofenil( $\left(1^{\prime}, 2^{\prime}\right.$-di-hidroxibenzeno) boronato, $p$-aminofenil[( $4^{\prime}$-nitro)-1' ' $^{\prime}$-di-hidroxibenzeno]boronato e $p$-nitrofenil[(4'-amino)-1',2'-di-hidroxibenzeno] boronato são os que apresentam as melhores primeira hiperpolarizabilidades estáticas $\beta(0)$ e dinâmicas $\beta(\omega)$, com valores calculados $(\mathrm{HF} / 6-31 \mathrm{G}++(d, p))$ da ordem de $20 \times 10^{-30}-30 \times 10^{-30}$ esu. $^{12}$

Ésteres boronatos são formados a partir de reações de condensação de dióis com boranos formando dialcoxiboranos, $\mathrm{H}\left[\mathrm{BO}_{2}\right] \mathrm{RR}^{\prime}$, que, ao reagirem com alcenos ou alcinos, conduzem aos derivados desejados, Esquema 1. Os ésteres boronatos são bons ácidos de Lewis, devido ao caráter vacante do orbital $2 p$ do átomo de boro trivalente. Esse orbital pode, também, interagir com os orbitais $p_{\pi}$ ou $p_{\mathrm{n}}$ dos átomos de carbono ou oxigênio adjacentes formando unidades conjugadas estendidas. ${ }^{13}$

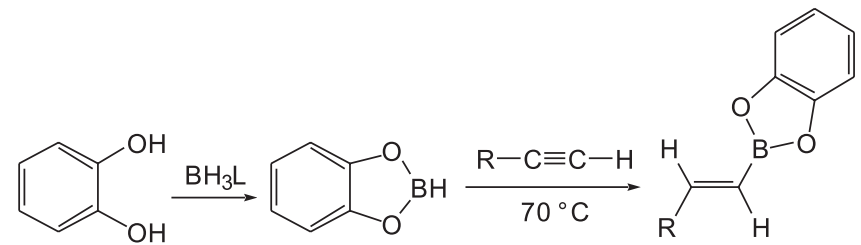

Esquema 1. Reação de formação dos catecóis boranos a partir de alcoxiboranos

Os elementos metálicos $\mathrm{n} p$ do grupo $\mathbf{3 A}$, alumínio $(\mathrm{n}=3)$ e gálio $(n=4)$, podem vantajosamente substituir o átomo de boro nos boronatos orgânicos, conferindo aos homólogos organoaluminatos ou organogaliatos um aumento comparativo nas suas propriedades óticas não-lineares. Os raios iônicos dos elementos $\mathrm{B}, \mathrm{Al}$ e $\mathrm{Ga}$ são, respectivamente, 0,$41 ; 0,675$ e $0,76 \AA$, e suas eletronegatividades, na escala de Pauling, são 1,61 (Al) e 1,81 (Ga), um pouco menor que 2,04, a eletronegatividade do átomo de boro. Como complemento, a polarizabilidade atômica desses elementos é 3,03 $\AA^{3}$ (B), 6,8 $\AA^{3}$ (Al) e $8,12 \AA$ (Ga). ${ }^{14}$ A partir dos valores dessas propriedades atômicas, pode-se esperar que os derivados contendo o átomo mais volumoso e mais polarizável do Ga apresentem polarizabilidades, e possivelmente as primeiras hiperpolarizabilidades, relativamente maiores que a dos seus análogos mais leves aluminatos e boronatos.

Motivados por essas expectativas, uma investigação teórica simples foi realizada para se determinar, por meio de cálculos $a b$ initio de estruturas eletrônicas Hartree-Fock, as polarizabilidades e primeira hiperpolarizabilidades de um conjunto de ésteres homólogos E-4-amino-trans-1,3-butadienil-(1',2'-di-hidroxibenzeno)boronato (B1), E-4-amino-trans-1,3-butadien-(1',2'-di-hidroxibenzeno)aluminato (Al1) e E-4-amino-trans-1,3-butadien-(1',2'-di-hidroxibenzeno) galiato (Ga1). Foram estudadas três séries de moléculas, duas delas contendo um grupo nitro nas posições 3' (B2, Al2, Ga2) ou 4' (B3, Al3, Ga3) do anel fenílico. O par de substituintes nitro e amino foram estrategicamente incluídos ao esqueleto básico do trans-1,3butadienil-(1',2'-di-hidroxibenzeno) para se ter uma estrutura análoga a um composto do tipo push-pull D-R e, consequentemente, boas hiperpolarizabilidades moleculares.

\section{PARTE EXPERIMENTAL}

A energia eletrônica de uma molécula $E(\mathbf{E})$ é alterada em resposta a uma perturbação de um campo elétrico externo estático $\mathbf{E}(0)$ ou dependente da freqüência $\mathbf{E}(\omega)$. Para campos elétricos relativamente fracos, essa energia pode ser expandida em uma série de Taylor nas componentes do campo aplicado, tal que,

$$
E(\mathbf{E})=E(\mathbf{0})+\boldsymbol{\mu} \cdot \mathbf{E}+\frac{1}{2} \boldsymbol{\alpha}: \mathbf{E}: \mathbf{E}+\frac{1}{6} \boldsymbol{\beta}: \mathbf{E}: \mathbf{E}: \mathbf{E}+\frac{1}{24} \gamma: \mathbf{E}: \mathbf{E}: \mathbf{E}: \mathbf{E}+\cdots
$$

Os coeficientes vetorial $\mu$ e tensoriais $\alpha, \beta, \gamma$ etc. na expansão acima definem, respectivamente, as propriedades moleculares elétricas, momento de dipolo permanente, polarizabilidade, primeira hiperpolarizabilidade, segunda hiperpolarizabilidade etc. Para campos elétricos externos dependentes do tempo (na forma de uma fase $\omega t$ ), a polarizabilidade e as hiperpolarizabilidades moleculares tornamse quantidades dependentes da frequiência $\omega$ do campo elétrico da perturbação. ${ }^{15-18}$

Dentre os objetos requeridos para a previsão $a b$-initio das (hiper) polarizabilidades moleculares estão um conjunto de base de funções atômicas adequadas, o tratamento de correlação eletrônica e a dependência dessas propriedades moleculares (dispersão) com a frequência da radiação eletromagnética aplicada. Neste trabalho, foram obtidas (hiper)polarizabilidades aproximadas utilizando-se de funções de onda Hartree-Fock não correlacionadas, expandidas em uma base de funções atômicas duplas na região de valência (double-zeta) mais funções difusas e de polarização. O emprego do método de cálculo não correlacionado se justifica, em parte, em razão de desejarmos, com estes estudos, respostas comparativas simples para as (hiper)polarizabilidades de moléculas homólogas contendo diferentes elementos do grupo 3A. A expectativa é que as diferenças dos efeitos da correlação eletrônica nas propriedades elétricas entre pares dessas moléculas sejam desprezíveis. Também, resultados anteriores apontam que as hiperpolarizabilidades de moléculas estendidas são relativamente menos dependentes da contribuição da correlação eletrônica e mais da qualidade do conjunto de bases atômicas empregadas. ${ }^{1}$

Todos os cálculos foram realizados para as funções de onda com uma simetria de pontos do grupo $C_{1}$. A orientação comum adotada para as todas as moléculas se fez tal que o segmento de linha reta que passa pela ligação $\mathrm{Z}-\mathrm{C}(\mathrm{Z}=\mathrm{B}, \mathrm{Al}, \mathrm{Ga})$ define o eixo $x$ e o plano base das moléculas coincide como o plano $x y$ de um sistema de coordenadas cartesiano, conforme ilustrado na Figura 1.

Nenhuma correção devido aos movimentos vibracionais ou para as contribuições devido aos possíveis efeitos de um meio solvente foi considerada. Essas correções, quando aplicáveis, são estimadas contribuir entre 5-10\% para os valores obtidos para as (hiper)polarizabilidades de moléculas pequenas isoladas. ${ }^{18-20}$

As geometrias moleculares foram otimizadas utilizando-se um conjunto de bases atômicas tipo 6-31G $(d, p)^{21}$ para os átomos de $\mathrm{B}, \mathrm{C}, \mathrm{N}$ e $\mathrm{O}\left(\alpha_{d}=0,8\right)$ e hidrogênio $\left(\alpha_{p}=1,1\right)$. Para os átomos de alumínio e gálio foram utilizados um conjunto equivalente 6-31G $(d, p)^{22}$ com o esquema de contração $\operatorname{Al}[16 s 10 p 1 \mathrm{~d} \| 4 s 3 p 1 d] \mathrm{e}$ $\mathrm{Ga}[22 s 16 p 5 d \| 5 s 4 p 3 d]$. Os cálculos das propriedades elétricas lineares e não-lineares foram realizados utilizando-se bases de funções atômicas do tipo 6-31++G $(d, p){ }^{21}$ Todas as estruturas moleculares foram otimizadas utilizando o conjunto de programas de cálculos de estruturas e propriedades eletrônicas GAMESS ${ }^{23}$ enquanto que os momentos de dipolo, polarizabilidades e hiperpolarizabilidades elétricas foram obtidas utilizando-se o método da teoria de resposta $^{17,18}$ implementada no programa DALTON. ${ }^{24}$ As (hiper)polarizabilidades foram calculadas para perturbações elétricas estáticas $\omega=0$ e dependentes da frequência de uma radiação eletromagnética. As polarizabilidades $\alpha(\omega)$ e primeira hiperpolarizabilidades $\beta(-2 \omega ; \omega, \omega)$ 
das moléculas de interesse deste trabalho foram calculadas para três frequências da radiação eletromagnética, 1064, 694 e 465 nm, que

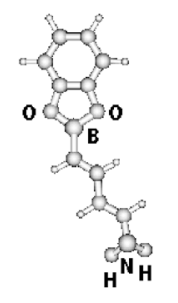

B1

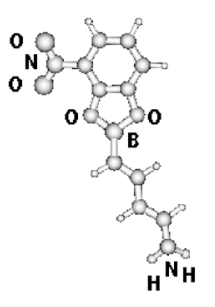

B2

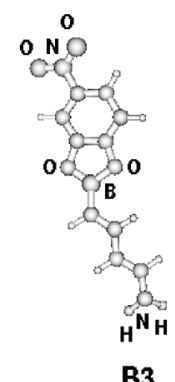

B3

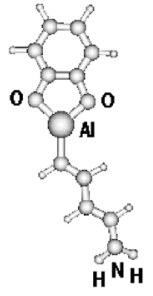

Al1

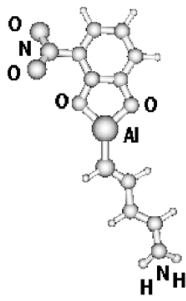

Al2

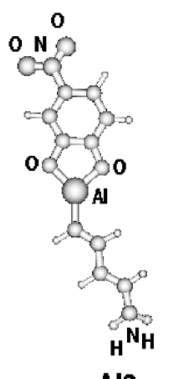

Al3
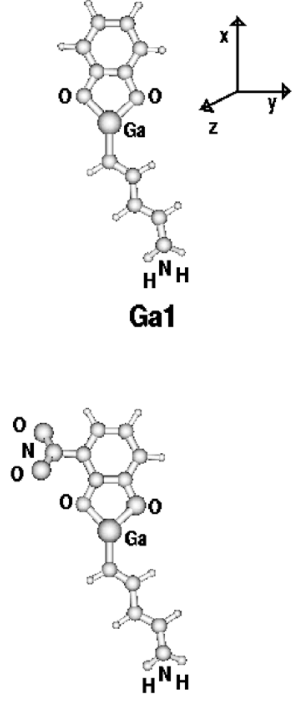

Ga2

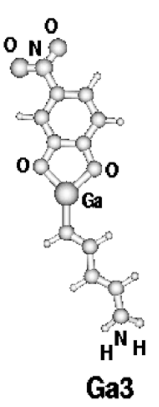

Figura 1. Estruturas moleculares otimizadas, $H F / 6-31 G(d, p)$ para as moléculas

E-4-amino-trans-1,3-butadienil-(1',2'-dihidroxibenzeno)boronato B1,

E-4-amino-trans-1,3-butadienil-(1',2'-dihidroxibenzeno)aluminato Al1,

E-4-amino-trans-1,3-butadienil-(1',2'-dihidroxibenzeno)galiato Ga1,

E-4-amino-trans-1,3-butadienil-(1',2'-dihidroxi-3'-nitrobenzeno)boronato $\mathbf{B 2}$,

E-4-amino-trans-1,3-butadienil-(1',2'-dihidroxi-3'-nitrobenzeno)aluminato

Al2,

E-4-amino-trans-1,3-butadienil-(1',2'-dihidroxi-3'-nitrobenzeno)galiato Ga2,

E-4-amino-trans-1,3-butbutadienil -(1',2'-dihidroxi-4'-nitrobenzeno)boronato B3,

E-4-amino-trans-1,3-butadienil-(1',2'-dihidroxi-4'-nitrobenzeno) aluminato Al3,

E-4-amino-trans-1,3-butadienil-(1',2'-di hidroxi-4'-nitrobenzeno) galiato $\mathbf{G a 3}$ correspondem às frequências de operação dos lasers $\mathrm{Nd}^{3+}$ :YAG, rubi e (o centro da banda de emissão) do laser do corante de cumarina, respectivamente. Os resultados ${ }^{12}$ mostram que $\alpha(\omega)$ e $\beta(-2 \omega ; \omega, \omega)$ aumentam muito lentamente com $\omega$ e, no que se segue, apenas as polarizabilidades $\alpha(0)$ e primeira hiperpolarizabilidades $\beta(0)$ estáticas serão referidas.

As polarizabilidades moleculares serão dadas para as suas componentes tensoriais e médias isotrópicas,

$$
\begin{aligned}
& \alpha_{\mathrm{av}}=\frac{1}{3}\left(\alpha_{x x}+\alpha_{y y}+\alpha_{z z}\right) \\
& \beta_{\mathrm{i}}=\sum_{j=x y z}\left(\beta_{i j j}+\beta_{j i j}+\beta_{j j i}\right), \quad j=x, y, z
\end{aligned}
$$

As quantidades $\beta_{i}(0)$ são conhecidas como as componentes vetoriais do tensor da primeira hiperpolarizabilidade. As projeções dessas componentes ao longo das direções do vetor momento de dipolo elétrico são parâmetros importantes em experimentos que requerem a orientação de uma molécula por um campo elétrico externo. ${ }^{25}$

\section{RESULTADOS E DISCUSSÃO}

\section{Estruturas e momentos de dipolo elétricos}

A estrutura base dos E-4-amino-trans-1,3-butadienil-(1',2'-dihidroxibenzeno), ou simplesmente amino-butadienil catecóis, contem o grupo funcional $\left[\mathrm{ZO}_{2}\right],(\mathrm{Z}=\mathrm{B}, \mathrm{Al}, \mathrm{Ga})$ central promovendo a conjugação de elétrons entre dois fragmentos insaturados. Três variedades desses compostos foram selecionadas para investigações $a b$-initio de suas polarizabilidades e primeira hiperpolarizabilidades elétricas. As estruturas moleculares dos catecóis boronatos, aluminatos e galiatos otimizadas pelo método Hartree-Fock e funções de onda 6-31G $(p, d)$ são apresentadas na Figura 1 e os principais parâmetros estruturais estão especificados na Tabela 1. Uma visão geral dessas estruturas é dada pelo produto da síntese esquemática apresentado no Esquema 1 , fazendo $\mathrm{R}=-\mathrm{CH}=\mathrm{CHNH}_{2}$.

Todas as moléculas mostram uma base estrutural plana em torno dos elementos $\mathrm{B}, \mathrm{Al}$ ou $\mathrm{Ga}$, enquanto que aquelas contendo o grupo amino mostram uma estrutura piramidal local em torno do átomo de nitrogênio. Os derivados 2'-nitro catecol de alumíno e gálio têm, ambos, o plano do grupo nitro alinhando-se fora do plano do anel aromático com um ângulo de torção de $\sim 28^{\circ}$. Claramente, observa-se a tendência esperada de um aumento entre as distâncias de equilíbrio $r_{\text {eq }}(\mathrm{C}-\mathrm{Z})$ e $r_{\text {eq }}(\mathrm{Z}-\mathrm{O})$ e os raios iônicos dos elementos $\mathrm{Z}$ do grupo $\mathbf{3 A}$ da tabela periódica, $r(\mathrm{~B})<r(\mathrm{Al})<r(\mathrm{Ga})$, assim como uma consequente diminuição do ângulo de ligação $\theta(\mathrm{OZO})$ que se aproxima de $90^{\circ}$ para moléculas contendo o átomo mais volumoso de gálio.

Os parâmetros geométricos calculados apresentam-se próximos dos valores relatados na literatura. Na literatura encontramos os catecóis boronatos, como o dicloreto $\mathrm{Cl}\left[\mathrm{BO}_{2} \mathrm{C}_{6} \mathrm{H}_{2} \mathrm{O}_{2} \mathrm{~B}\right] \mathrm{Cl}^{26}$ ou fenil--, ${ }^{27}$ $p$-toluil-28 ou mesmo estiril-29 catecol boronatos, com as distâncias

Tabela 1. Distâncias de equilíbrio $r_{\text {eq }}$ (em angström) e ângulos de ligação (em graus) calculados para os aminobutadienil catecóis boronatos B1-B3, aluminatos Al1-A13 e galiato Ga1-Ga3

\begin{tabular}{lcrrrrrrrr}
\hline Molécula & B1 & Al1 & Ga1 & B2 & Al2 & Ga2 & B3 & Al3 & Ga3 \\
\hline$r_{e q}(\mathrm{ZO})^{\mathrm{a}}$ & 1,38 & 1,73 & 1,81 & 1,39 & 1,74 & 1,81 & 1,39 & 1,74 & 1,82 \\
$r_{e q}(\mathrm{CZ})$ & 1,54 & 1,90 & 1,91 & 1,53 & 1,90 & 1,90 & 1,53 & 1,90 & 1,90 \\
$\theta(\mathrm{OZO})$ & 110 & 96 & 92 & 109 & 94 & 91 & 109 & 94 & 91 \\
$\theta(\mathrm{ZOC})^{\mathrm{a}}$ & 106 & 107 & 107 & 107 & 108 & 109 & 106 & 108 & 108 \\
\hline
\end{tabular}

(a) Valores médios. $\mathrm{Z}=\mathrm{B}, \mathrm{Al}, \mathrm{Ga}$. 
Tabela 2. Momento de dipolo elétrico (em unidades de Debye, 1 Debye $=10^{-18} \mathrm{esu}$ ), polarizabilidade elétrica (em unidades de $10^{-24} \mathrm{~cm}^{3}$ ) para os aminobutadienil catecóis boronatos B1-B3, aluminatos Al1-Al3 e galiatos Ga1-Ga3, calculados para funções de onda Hartree-Fock e um conjunto de base atômicas 6-31++G(d,p)

\begin{tabular}{lcccccccc}
\hline & $\mu_{x}$ & $\mu_{y}$ & $\|\mu\|^{\mathrm{a}}$ & $\alpha_{x x}$ & $\alpha_{y v}$ & $\alpha_{z y}$ & $\alpha_{a v}$ \\
\hline B1 & $-2,543$ & $-2,013$ & 3,401 & 215,2 & 149,5 & 78,4 & 147,7 & 44,3 \\
Al1 & $-5,921$ & $-2,167$ & 6,374 & 223,6 & 156.0 & 85,6 & 155,1 \\
Ga1 & $-7,318$ & $-2,342$ & 7,735 & 236,1 & 156,5 & 85,7 & 159,4 \\
B2 & -3.956 & -7.331 & 8.386 & 239,1 & 174,5 & 82,0 & 165,2 & 48,8 \\
Al2 & $-7,747$ & $-7,385$ & 10,737 & 243,8 & 179,3 & 92,4 & 171,8 \\
Ga2 & $-9,223$ & $-7,641$ & 12,004 & 245,3 & 165,9 & 63,6 & 158,3 \\
B3 & $-8,025$ & $-4,923$ & 9,462 & 244,7 & 167,9 & 81,5 & 164,7 & 47,7 \\
Al3 & $-11,726$ & $-4,868$ & 12,725 & 256,1 & 173,7 & 88,5 & 172,8 & 51,5 \\
Ga3 & $-13,266$ & $-5,046$ & 14,215 & 269,1 & 175,0 & 88,6 & 177,6 & 52,7 \\
\hline
\end{tabular}

(a) $\|\mu\|=\left(\mu_{x}^{2}+\mu_{y}^{2}+\mu_{z}^{2}\right)^{1 / 2}$

e ângulos de ligação $\mathrm{B}-\mathrm{O}$ e $\theta(\mathrm{OBO})$ determinadas experimentalmente em torno de $1,39 \AA$ e $111^{\circ}$, respectivamente. As distâncias de ligação B-C medidas experimentalmente são da ordem de 1,533 A. ${ }^{27-29}$ Da mesma forma, encontramos nos aluminanos trivalentes, como o $\mathrm{Al}(\mathrm{OAr})_{3}$, a distância de ligação $r(\mathrm{Al}-\mathrm{O})$ em torno de 1,65 $\AA$, e nos galianos do tipo $\beta-\mathrm{Ga}_{2} \mathrm{O}_{3}$ as distâncias de ligação $r(\mathrm{Ga}-\mathrm{O})$ da ordem de $1,83 \AA{ }^{26}$

Todas as estruturas moleculares otimizadas representam geometrias de equilíbrio autênticas, com todas as frequências vibracionais harmônicas reais. Em particular, os modos normais de vibração correspondentes aos modos de estiramento $v_{1}(\mathrm{CZ})$, estiramentos simétrico $\mathrm{v}_{2 \mathrm{~S}}\left(\mathrm{ZO}_{2}\right)$ e assimétrico $\mathrm{v}_{2 \mathrm{~A}}\left(\mathrm{ZO}_{2}\right)$ foram calculados com os valores (não escalonados) de $v_{1}(\mathrm{CB})=906, v_{1}(\mathrm{CAl})=841, v_{1}(\mathrm{CGa})$ $=807, v_{2 \mathrm{~S}}\left(\mathrm{BO}_{2}\right)=894, \mathrm{v}_{2 \mathrm{~S}}\left(\mathrm{AlO}_{2}\right)=943, \mathrm{v}_{2 \mathrm{~S}}\left(\mathrm{GaO}_{2}\right)=885, \mathrm{v}_{2 \mathrm{~A}}\left(\mathrm{BO}_{2}\right)$ $=1392, \mathrm{v}_{2 \mathrm{~A}}\left(\mathrm{AlO}_{2}\right)=855 \mathrm{e}_{2 \mathrm{~A}}\left(\mathrm{GaO}_{2}\right)=777 \mathrm{~cm}^{-1}$, para os compostos amino derivados mais simples B1, Al1 e Ga1, respectivamente.

As estruturas moleculares dos aminobutadienil catecóis geram distribuições de cargas que produzem os momentos de dipolo elétricos permanentes apresentados na Tabela 2. A componente longa $\mu_{x}$ dos momentos de dipolo elétricos de todas as moléculas tem a carga negativa apontando na direção ao grupo amino. Isso indica que os centros borato $\left[\mathrm{BO}_{2}\right]$, aluminato $\left[\mathrm{AlO}_{2}\right]$ e galiatos $\left[\mathrm{GaO}_{2}\right]$ atuam como grupos doadores eficientes de elétrons. Esses grupos são, por outro lado, receptores de elétrons $\pi$. A habilidade doadora de elétrons desses grupos aparentemente aumenta com o aumento do número quântico principal $n$ do elemento central $n \mathrm{p}^{1} ;(\mathrm{B}(n=2), \mathrm{Al}(n=3), \mathrm{Ga}(n=4))$, em uma relação inversa com as suas eletronegatividades de Pauling. Assim, as componentes dos momentos de dipolo longitudinal do conjunto das moléculas homólogas $\mathbf{Z 1}, \mathbf{Z 2}, \mathbf{Z 3}$, variam na ordem (Ga1) $>($ Al1 $)>($ B1 $) ;($ Ga2 $)>($ Al2 $)>($ B2 $)$ e $($ Ga3 $)>($ Al3 $)>($ B3 $)$. A inclusão de um grupo nitro no anel aromático causa um aumento nos momentos de dipolo da ordem de duas vezes (três vezes no caso dos catecóis boronato), com o melhor efeito visto para os derivados com a substituição 4'-nitro (substituição “meta”). A componente do transversal momento de dipolo $\mu_{y}$, coplanar ao plano da molécula, e causada principalmente pela disposição assimétrica do fragmento E-4-amino-trans-butadienila e das substituições 3 ou 4 do grupo nitro, nos derivados nitrocatecóis. A configuração piramidal do grupo amino e a torção estabilizadora do grupo nitro, observada nos derivados 3'nitrocatecol de alumínio e gálio, induzem uma pequena contribuição no momento de dipolo $\mu_{\mathrm{z}}$ na direção perpendicular do plano da molécula, que soma algo da ordem de $1 \mathrm{D}$.

\section{Polarizabilidades elétricas dipolares}

As polarizabilidades elétricas dipolares dos aminobutadienil catecóis, apresentadas na Tabela 2, por força de suas estruturas moleculares, mostram um alto grau de assimetria, $\operatorname{com} \alpha_{x x} \approx(3 / 2) \alpha_{y y} \approx 3 \alpha_{z z}$, e um valor médio $\alpha_{\text {av }}$ que se aproxima da componente transversal no plano $\alpha_{y y}$. Devido à escolha da orientação do sistema de coordenadas cartesiano de referência, as componentes fora da diagonal $\alpha_{x z}$ e $\alpha_{y z}$ do tensor de polarizabilidade são todas desprezíveis, enquanto que a componente fora da diagonal no plano $\alpha_{x y}$ tem um valor da ordem da metade do valor das menores componentes diagonais, $\alpha_{z z}$.

A introdução do grupo nitro no anel aromático afeta modestamente as polarizabilidades do grupo dessas moléculas independente do átomo 3A central de ligação. É visto, também, que um substituinte nitro na posição 4’ do anel aromático favorece uma polarizabilidade com um valor ligeiramente maior que a correspondente determinada para a substituição em 3'. As polarizabilidades moleculares variam muito lentamente com a troca dos elementos do grupo $\mathbf{3 A}$, sendo observada uma diferença da ordem de $10 \%$ entre os valores máximos e mínimos da polarizabilidade média $\alpha_{a v}$ correspondentes aos catecóis boronatos e galiatos.

Nenhuma relação quantitativa simples é observada entre $\alpha_{\mathrm{av}} \mathrm{e}$ as polarizabilidades atômicas do B, $\mathrm{Al}$ e $\mathrm{Ga}$ ou entre as distâncias interatômicas $\mathrm{C}-\mathrm{Z}$ ou $\mathrm{Z}-\mathrm{O}$. Isso indica que as polarizabilidades moleculares dos compostos estudados não podem ser entendidas como uma propriedade aditiva simples, formadas a partir das polarizabilidades dos fragmentos que as definem, os cromóforos conjugados aminobutadienila e catecoila e os átomos pivô B, Al e Ga. Espera-se que efeitos cooperativos entre as polarizabilidades de grupo atuem decisivamente para a formação das polarizabilidades das unidades moleculares. Investigações equivalentes foram relatadas na literatura para a dependência das polarizabilidades (e primeira hiperpolarizabilidade) elétricas dos heterociclos de cinco membros $\mathrm{C}_{4} \mathrm{H}_{6} \mathrm{Z}$ Para $\mathrm{Z}=(\mathrm{BH}, \mathrm{AlH}), \mathrm{Z}=\left(\mathrm{CH}_{2}, \mathrm{SiH}_{2}\right), \mathrm{Z}=(\mathrm{NH}, \mathrm{PH})$ e $\mathrm{Z}=(\mathrm{O}, \mathrm{S})$ cálculos teóricos foram realizados para funções de onda Hartree-Fock e, em alguns casos, com a inclusão dos efeitos de correlação através de correções MP2. ${ }^{30}$ Também, resultados DFT (DFT mais pseudo-potencial efetivo, para as moléculas contendo átomos pesados) foram apresentados para os heterociclos de cinco membros $\mathrm{C}_{4} \mathrm{H}_{4} \mathrm{Z}$ contendo os elementos da família $\mathbf{5 A}, \mathrm{Z}=\mathrm{N}, \mathrm{P}, \mathrm{As}, \mathrm{Sb}, \mathrm{Bi} .{ }^{31}$ Os resultados mostram que as polarizabilidades (e primeira hiperpolarizabilidades) moleculares aumentam uniformemente, ao longo de uma mesma família, para a substituição do heteroátomo mais pesado. Nesses trabalhos foram também exploradas possíveis correlações existentes entre a polarizabilidade elétrica e a eletronegatividade atômica de Pauling do heteroátomo e no parâmetro geométrico correspondente à distância de equilíbrio Z-C encontradas para os anéis de cinco membros.

As polarizabilidades dos aminobutadienil catecóis B1-B3, Al1-Al3 e Ga1-Ga3 têm origem na facilidade com que a densidade 
eletrônica é deformada, sob ação de um campo elétrico perturbativo, causado pela transferência de cargas entre as unidades catecoila e aminobutadienila, unidos entre si pelos átomos de. B, Al ou Ga. Uma inspeção nos primeiros orbitais de fronteira dessas moléculas, Figuras2-4, é esclarecedora nesses aspectos. Basicamente, os orbitais HOMO, HOMO-1 e HOMO-2 correspondem aos orbitais ocupados de maior energia, grandemente localizados sobre os fragmentos aminobutadienlila ou catecoila. Alguns desses orbitais contêm contribuições importantes de amplitudes eletrônicas sobre o grupo amino. Por outro lado, os orbitais LUMO (LUMO+1 em B2 e B3) mostram sistematicamente uma grande amplitude eletrônica na forma de um orbital ligante sobre os centros $\mathrm{CZ}(\mathrm{Z}=\mathrm{B}, \mathrm{Al}, \mathrm{Ga})$, e que contribui, assim, para o aumento da deslocalização eletrônica interfragmentos, aminobutadienila e catecoila. Nos derivados nitrocatecóis, os orbitais LUMO ou LUMO+1 carregam uma amplitude eletrônica apreciavelmente localizada sobre o grupo nitro. Do ponto de vista da expressão que permite o cálculo de polarizabilidade elétrica como uma somasobre-estados quânticos. ${ }^{1,2}$ As principais contribuições que entram na composição da polarizabilidade eletrônicas são devidas aos grupos de excitações eletrônicas $\mathrm{HOMO} \rightarrow \mathrm{LUMO}, \mathrm{HOMO} \rightarrow(\mathrm{LUMO}+1)$, $($ HOMO-1) $\rightarrow$ LUMO e (HOMO-1) $\rightarrow$ (LUMO+1) associadas com as menores energias de transição $\left(E=E_{\mathrm{m}}-E_{\mathrm{g}}\right)$ se dotadas, obviamente, de amplitudes de transições apreciáveis. No caso dos aminobutadienil catecóis de interesse, as transições HOMO $\rightarrow$ LUMO ou $\mathrm{HOMO} \rightarrow$ LUMO+1, com. energias de excitações da ordem de 4,5$6,5 \mathrm{eV}$, introduzem uma polarização efetiva intra e interfragmentos equivalentes para os derivados de boro, alumínio ou gálio. Espera-se que essas transições contribuam dominantemente para a formação das polarizabilidades elétricas desses compostos. Esses resultados devem ser entendidos como informações parciais, contudo, visto que as correções incluídas com o uso de uma base de funções atômicas mais difusas e melhor polarizadas e, com um menor peso, os efeitos devidos à correlação eletrônica podem alterar quantitativamente os valores obtidos para as polarizabilidade $\mathrm{HF} / 6-311++\mathrm{G}(d, p)$ aqui relatadas. Porém, pode-se estabelecer que, aparentemente, a vacância orbital $n \mathrm{p}$, $n=2,3,4$ dos átomos $\mathrm{B}, \mathrm{Al}$ ou $\mathrm{Ga}$, contribui aproximadamente de forma equivalente na composição dos orbitais de fronteira HOMO-LUMO conferindo, assim, uma polarizabilidade equivalente aos derivados aminobutadienil catecóis contendo esses átomos centrais.

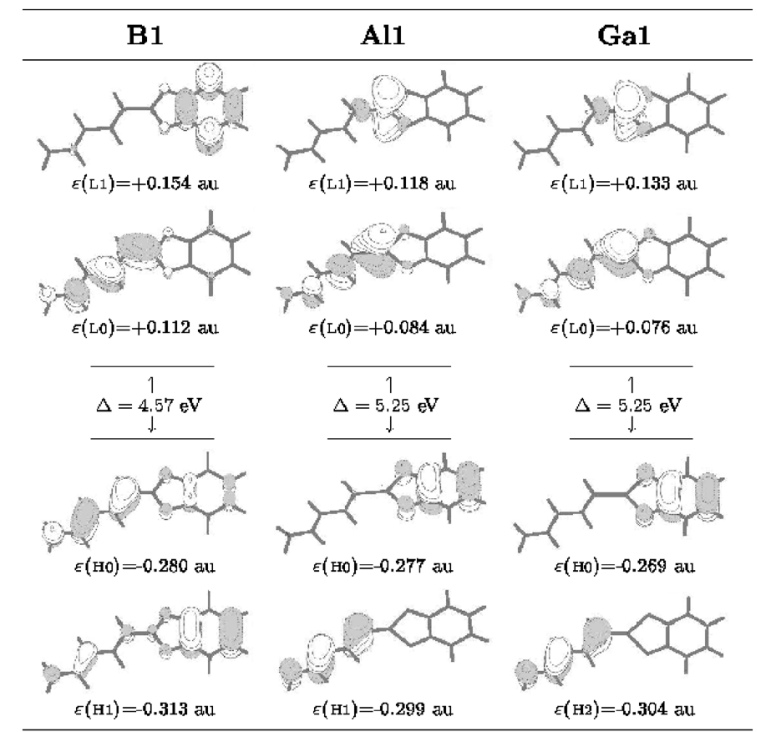

Figura 2. Primeiros orbitais de fronteira dos ésteres B1, Al1 $e \mathbf{G a 1}$ e suas diferenças de energias orbitais HOMO-LUMO

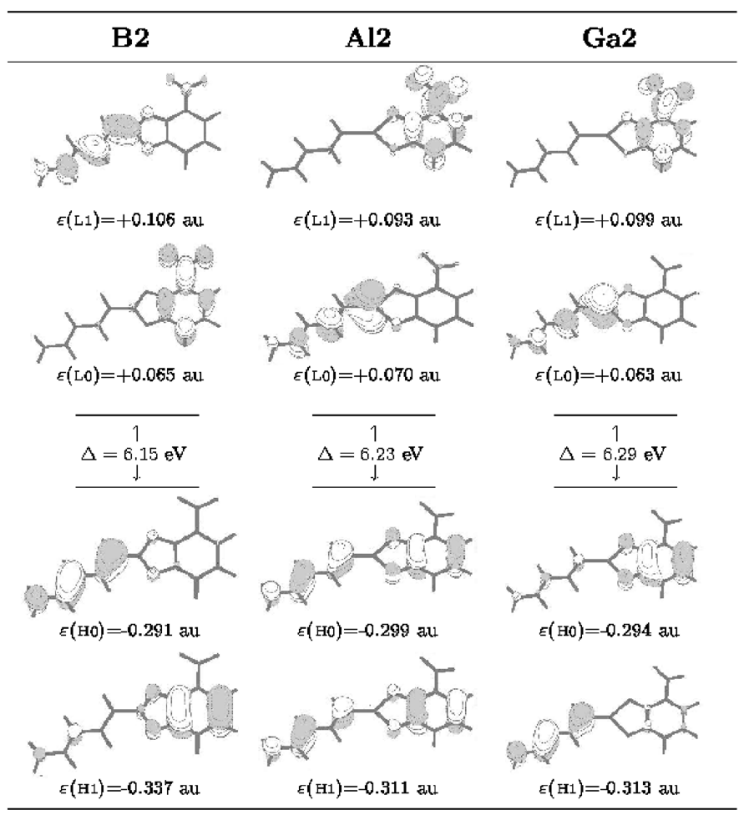

Figura 3. Primeiros orbitais de fronteira dos ésteres $\mathbf{B 2}, \mathbf{A 1 2} e \mathbf{G a 2}$ e suas diferenças de energias orbitais HOMO-LUMO

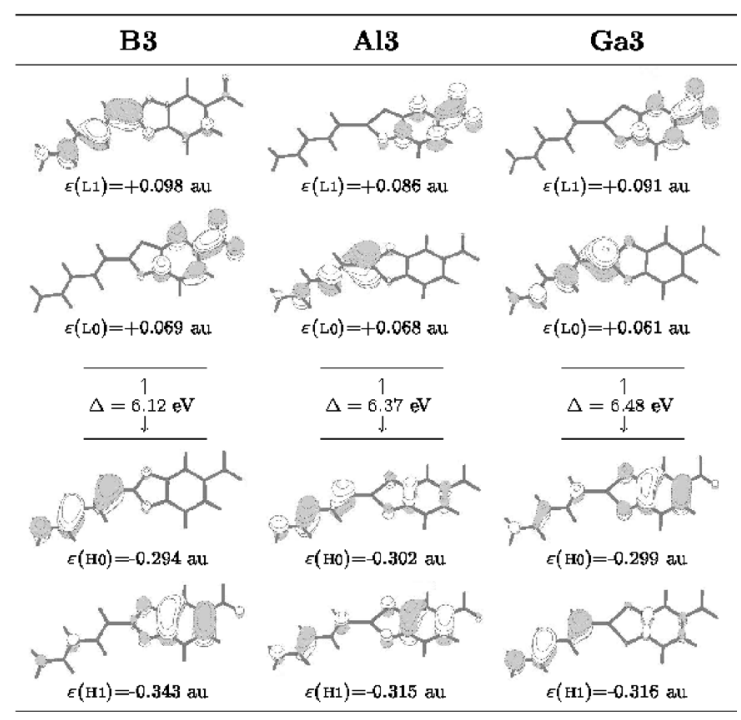

Figura 4. Primeiros orbitais de fronteira dos ésteres $\mathbf{B 3}, \mathbf{A l 3} e \mathbf{G a 3}$ e suas diferenças de energias orbitais HOMO-LUMO

\section{Primeira hiperpolarizabilidades elétricas dipolares}

Em um dos primeiros estudos teóricos realizados, Oudar e Chemla ${ }^{25}$ mostraram que a variação na posição relativa dos grupos substituintes amino e nitro, posições orto $(1,2)$, meta $(1,3)$ ou para $(1,4)$ no anel fenólico da nitroanilina influencia consideravelmente os valores das suas polarizabilidades e primeira hiperpolarizabilidades. Analogamente pode-se esperar que os isômeros nitro substituídos B2 e B3, Al2 e Al3 e Ga2 e $\mathbf{G a 3}$ apresentem polarizabilidades e hiperpolarizabilidades diferenciadas. É antecipado que os isômeros contendo o grupo nitro na posição $4^{\prime}$ do anel catecoíla apresente melhores resultados para as primeiras hiperpolarizabilidades $\beta(0)$, devido à formação de uma incipiente estrutura do tipo push-pull D-R. Assim, pode-se formular que as moléculas dos compostos B2, 
Al2 e Ga2, e, particularmente, B3, Al3 e Ga3 podem ser vistas como formadas por dois pares de unidades eletrônicas $\mathrm{D}-\mathrm{R}$ dispostos em fase, com o grupo [ZO2] ( $\mathrm{Z}=\mathrm{B}, \mathrm{Al}, \mathrm{Ga})$ chaveando o fluxo da densidade eletrônica entre as extremidades doadora $\left(\mathrm{NH}_{2}\right)$ e retiradora $\left(\mathrm{NO}_{2}\right)$ de elétrons.

As componentes $\beta_{x}(0)$ e $\beta_{y}(0)$, no plano base das moléculas B1B3, Al1-Al3 e Ga-Ga3 estão apresentadas na Tabela 3. A contribuição de $\beta_{z}(0)$, para cada um dos compostos estudados, é pequena e tem um valor típico da ordem de $1 \%$ do valor da componente longitudinal $\beta(0)$. Como antecipado, a primeira hiperpolarizabilidade desses derivados aumenta suavemente com a introdução do grupo nitro no anel catecoíla e, esse efeito, é maior para a substituição na posição 4" ("orto"). Uma exceção é notada, entretanto, entre as hiperpolarizabilidades dos derivados de gálio Ga1 e Ga2 que, essencialmente, possuem os mesmos valores para as hiperpolarizabilidades moleculares calculadas.

Os resultados encontrados para as hiperpolarizabilidades dos aminobutadienila catecóis comparam-se aos valores relatados para a $p$-nitroanilina, (em módulo) 4,40×10 $0^{-32}$ esu de um cálculo HartreeFock empregando um conjunto de bases atômicas do tipo DZV $+2 d,{ }^{32}$ e $15,74 \times 10^{-32}$ esu, para cálculos correlacionados DFT/B3LYP ${ }^{33,34}$ usando um conjunto de bases atômicas desenvolvido por Sadlej. ${ }^{35}$ Para efeitos comparativos, utilizando a mesma metodologia e funções de base atômicas empregadas nos cálculos das hiperpolarizabilidades das moléculas dos aminobutadienila catecóis, obtemos o valor de $\beta_{x}(0)=6,34 \times 10^{-32}$ esu para a $p$-nitroanilina. Isto significa que os derivados aminobutadienila catecóis têm uma eficiência prevista da ordem de 2 a 3 vezes maiores que a $p$-nitroanilina para um processo ótico não-linear, como, por exemplo, a geração de um segundo harmônico. Não raramente, não é imediata uma comparação direta de hiperpolarizabilidades moleculares calculadas teoricamente e aquelas determinadas por meio de métodos experimentais diferentes. Uma discussão sobre esse ponto foi apresentada por Willetts et al. ${ }^{36}$ e re-examinada recentemente por Reis, ${ }^{37}$ que apresenta exemplos de valores experimentais obtidos por diferentes métodos computacionais $a b$-initio e experimentais para a primeira hiperpolarizabilidade da $p$-nitroanilina.

Diferentemente da tendência apresentada para as polarizabilidades, as primeiras hiperpolarizabilidades dos derivados B1-B3, Al1-Al3 e Ga1-Ga3 aumentam (em módulo) com a substituição do átomo de boro por alumínio, mas reduzem se uma nova substituição do átomo de alumínio pelo gálio for feita; as relações $\beta_{x}(\mathbf{A l 1})>$ $\beta_{x}(\mathbf{G a l})>\beta_{x}(\mathbf{B 1}) ; \beta_{x}(\mathbf{A l 2})>\beta_{x}(\mathbf{B} 2)>\beta_{x}(\mathbf{G a 2})$ e $\beta_{x}(\mathbf{A l 3})>\beta_{x}(\mathbf{G a 3})$ $>\beta_{x}$ (B3) são agora observadas. Esse comportamento pode ser prontamente entendido se observarmos as hiperpolarizabilidades calculadas $(\mathrm{HF} / 631++\mathrm{G}(d, p)), \beta_{x}(0)[\mathbf{B} \mathbf{0}]=-1,79 \times 10^{-32}$ esu, $\beta_{x}(0)[\mathbf{A l 0}]=$ $-0,45 \times 10^{-32}$ esu e $\beta_{x}(0)[\mathbf{G a 0}]=+0,88 \times 10^{-32}$ esu, para as moléculas dos derivados não substituídos do benzodioxoboro B0 $\left(\mathrm{H}\left[\mathrm{BO}_{2}\right]\right.$ $\left.\mathrm{C}_{6} \mathrm{H}_{4}\right)$, benzodioxoalumínio $\mathbf{A l 0}\left(\mathrm{H}\left[\mathrm{AlO}_{2}\right] \mathrm{C}_{6} \mathrm{H}_{4}\right)$ e benzodioxogálio $\mathbf{G a 0}\left(\mathrm{H}\left[\mathrm{GaO}_{2}\right] \mathrm{C}_{6} \mathrm{H}_{4}\right)$. Como destaque, vemos a suave diminuição dos valores de $\beta_{x}(0)$ nesta série, que culmina com a troca de sinal para o derivado de Ga. Essa mesma tendência pode ser verificada nos valores das hiperpolarizabilidades da série Al1-Ga1, Al2-Ga2 e Al3-Ga3, quando $\beta_{x}(0)$ dos derivados de Ga tornam-se menos negativos que os correspondentes dos homólogos de Al. A polarização introduzida pelo grupo aminobutadienila compensa a redução do valor de $\beta_{x}(0)$ para os pares B1-Al1, B2-Al2 e B3-Al3, se comparados com B0-Al0.

Deve-se notar o sinal negativo da componente vetorial $\beta_{x}(0)$ calculada para todos os derivados aminobutadienil catecoíla. Essa mesma tendência é também vista nas hiperpolarizabilidades de heterociclos de cinco membros $\mathrm{C}_{4} \mathrm{H}_{4} \mathrm{Z} ; \mathrm{Z}=\mathrm{N}, \mathrm{P}, \mathrm{As}, \mathrm{Sb}, \mathrm{Bi}$, como calculadas por Alparone et al.. ${ }^{31}$ De acordo com um modelo de dois estados, ${ }^{2,38}$ a componente vetorial da primeira hiperpolarizabilidade pode ser estimada pela relação

$\beta(0) \propto \frac{\left(\mu_{m m}-\mu_{g g}\right)\left|\mu_{g m}\right|^{2}}{\left(E_{g}-E_{m}\right)^{2}}$

Nesta aproximação, a soma sobre todos os estados quânticos excitados é restrita a um único estado excitado, $\beta(0)$ mostra uma dependência no quadrado do momento de dipolo de transição $\mu_{g m}$ e da diferença $\Delta_{\mu}$ entre os momentos de dipolo permanentes $\mu_{m m}$ e $\mu_{g g}$ dos estados fundamental $g$ e excitado $m$. O sinal negativo para uma componente vetorial da hiperpolarizabilidade é, então, interpretado pela diferença negativa de $\Delta_{\mu}$ para as componentes cartesianas de interesse dos respectivos momentos de dipolo. No nosso caso, $\left(\mu_{x}\right)$ ${ }_{g g}$ são quantidades negativas, Tabela 2, e, portanto, espera-se que os momentos de dipolo $\left(\mu_{x}\right)_{m m}$ do estado excitado relevante (possivelmente um do primeiros estados excitados) de todos os aminobutadienil catecóis considerados tenham um sinal negativo e, em módulo, maiores que $\left(\mu_{x}\right)_{g g}$. Finalmente, pode-se inferir que, observando que os orbitais LUMO ou LUMO+1 de $\mathbf{Z 2 - Z 3 ~ ( Z = B , A l , G a ) ~ r e c e b e m ~}$ uma grande amplitude de probabilidade sobre o grupo nitro, seus isômeros formados pela inversão entre as posições dos grupos nitro e amino, isto é, os derivados E-4-nitro-trans-1,3-butadienil-(1',2'di-hidroxi-3'-aminobenzeno)boronato, aluminato ou galiato, ou

Tabela 3. Componentes $\beta_{\mathrm{ijk}}$ e a parte vetorial $\beta_{\mathrm{i}}$ da primeira hiperpolarizabilidade (em unidades de $10^{-32}$ esu) para os aminobutadienil catecóis boronatos B1-B3, aluminatos Al1-Al3 e galiatos Ga1-Ga3, calculados para funções de onda Hartree-Fock e um conjunto de bases atômicas $6-31++\mathrm{G}(d, p) .1 \mathrm{esu}=3,71134 \times 10^{-21} \mathrm{C}^{3} \mathrm{~m}^{3} \mathrm{~J}^{-2}$

$\beta_{i=x, y, 2}=\frac{1}{3} \sum_{j}\left(\beta_{i j}+\beta_{j i j}+\beta_{j i j}\right)$

\begin{tabular}{lcccccccc}
\hline & $\beta_{x x x}$ & $\beta_{x y y}$ & $\beta_{x z z}$ & $\beta_{x}$ & $\beta_{y x x}$ & $\beta_{y y y}$ & $\beta_{y z z}$ & $\beta_{y}$ \\
\hline B1 & $-11,9$ & $-0,6$ & $-1,2$ & $-13,7$ & $-2,9$ & $+0,4$ & $-0,3$ & $-2,8$ \\
Al1 & $-13,1$ & $-1,2$ & $-1,8$ & $-16,1$ & $-3,8$ & $+0,1$ & $-0,5$ & $-4,2$ \\
Ga1 & $-12,2$ & $-1,1$ & $-1,6$ & $-14,9$ & $-3,8$ & $+0,2$ & $-0,5$ & $-4,0$ \\
B2 & $-14,9$ & $-0,9$ & $-0,9$ & $-16,7$ & $-3,5$ & $-0,8$ & $+0,1$ & $-4,2$ \\
A12 & $-15,1$ & $-1,4$ & $-1,5$ & -18.0 & $-4,0$ & -1.0 & $+0,0$ & $-5,0$ \\
Ga2 & $-13,1$ & $-0,9$ & $-0,7$ & $-14,8$ & $-3,9$ & $-0,4$ & $-0,3$ & $-4,7$ \\
B3 & $-16,5$ & $-1,3$ & $-0,5$ & $-18,2$ & $-5,9$ & $+1,0$ & 0,0 & -5.0 \\
A13 & $-18,1$ & $-2,0$ & $-1,0$ & $-21,1$ & $-7,4$ & $+0,8$ & $-0,3$ & $-6,8$ \\
Ga3 & $-18,0$ & $-2,0$ & $-0,8$ & $-20,8$ & -2.0 & $+0,9$ & $-0,2$ & $-1,2$ \\
\hline
\end{tabular}


E-4-nitro-trans-1,3-butadienil-(1',2'-di-hidroxi-4'-aminobenzeno) boronato, aluminato ou galiato terão ainda $\left(\mu_{x}\right)_{g g}$ negativos, mas $\left(\mu_{x}\right)$ ${ }_{m m}$ positivos. Assim, $\Delta_{\mu}$ será um parâmetro positivo e grande, o que implica que essas moléculas devem gerar as melhores primeira hiperpolarizabilidades $\beta_{\mathrm{x}}(0)$ para esta classe de compostos.

\section{AGRADECIMENTOS}

M. B. Procópio agradece ao Conselho Nacional de Desenvolvimento Científico e Tecnológico (CNPq), Brasil.

\section{REFERÊNCIAS}

1. Kanis, D. R.; Ratner, M. A.; Marks, T. J.; Chem. Rev. (Washington, DC, U.S.) 1994, 94, 195.

2. Parasad, P. N.; Williams, D. J.; Introduction to Nonlinear Optical Effects in Molecules and Polymers, John Wiley \& Sons: New York, 1991.

3. Garito, A.; Shi, R. F.; Wu, M.; Phys. Today 1994, 47, 51.

4. Hasanein, A. A.; Adv. Chem. Phys. 1993, 85 (Modern Nonlinear Optics, parte 2), 415.

5. Um exemplo de uma lista não exaustiva: Calabrese, J. C.; Cheng, L-T.; Green, J. C.; Marder, S. R.; Tamt, W.; J. Am. Chem. Soc. 1991, 113, 1221; Marder, S. R.; Perry, J. W.; Tiemann, B. G.; Organometallics 1991, 10, 1896; Kanis, D. R.; Ratner, M. A.; Marks, T. J.; J. Am. Chem. Soc. 1992, 114, 10338; Kanis, D. R.; Lacroix, P. G.; Ratner, M. A.; Marks, T. J.; J. Am. Chem. Soc. 1994, 116, 10089; Long, N. J.; Angew. Chem., Int. Ed. 1995, 34, 21; Alain, V.; Forth, A.; Barzoukas, M.; Chen, C-T.; Blanchard-Desce, M.; Marder, S. R.; Perry, J. W.; Inorg. Chim. Acta 1996, 242, 43; Barlow, S.; Bunting, H. E.; Ringhan, C.; Green, J. C.; Bublitz, G. U.; Boxer, S. G.; Perry, J. W.; Marder, S. R.; J. Am. Chem. Soc. 1999, 121, 3715; Balavoine, G. G. A.; Daran, J-C.; Iftime, G.; Lacroix, P. G.; Manoury, E.; Delaire, A.; Maltey-Fanton, I.; Nakatani, K.; Di Bella, S.; Organometallics 1999, 18, 21; Barlow, S.; Marder, S. R.; Chem. Commun. (Cambridge, U.K) 2000, 1555; Asselberghs, I.; Clays, K.; Persoons, A.; Wardb, M. D.; McCleverty; J., J. Mater. Chem. 2004, 14, 2831.

6. Yuan, Z.; Taylor, N. J.; Marder, T. B.; Williams, I. D.; Kurtz, S. K.; Cheng, L.-T.; Chem. Commun. (Cambridge, U.K) 1990, 21, 1489; Yuan, Z.; Taylor, N. J.; Sun, Y.; Marder, T. B.; Williams, D.; Cheng, L.-T.; J. Organomet. Chem. 1993, 449, 27; Yuan, Z.; Collings, J. C.; Taylor, N. J.; Marder, T. B.; Jardin, C.; Halet, J-F.; J. Solid State Chem. 2000, 154, 5; Yuan, Z.; Entwistle, C. D.; Collings, J. C.; Albesa-Jovè, D.; Batsanov, A. S.; Howard, J. A. K.; Taylor, J.; Kaiser, H. M.; Kaufmann, D. E.; Poon, S-Y.; Wong, W-Y.; Jardin, C.; Fathallah, S.; Boucekkine, A.; Halet, J-F.; Marder, T. B.; Chem. Eur. J. 2006, 12, 2758; Lequan, M.; Lequan, M.; Ching, K. C.; J. Mat. Chem. 1991, 1, 997; Lequan, M.; Lequan, R. M.; Ching, K. C.; Barzoukas, M.; Fort, A.; Lahoucine, H.; Bravic, G.; Chasseua, D.; Gaultier, J.; J Mat. Chem. 1992, 2, 719; Branger, C.; Lequan, M.; Lequan, R. M.; Barzoukas, M.; Fort, A.; J. Mat. Chem, 1996, 6, 555; Branger, C.; Lequan, M.; Lequan, R. M.; Large, M.; Kajzar, F.; Chem. Phys. Lett. 1997, 272, 265; Entwistle, C. D.; Marder, T. B.; Angew. Chem., Int. Ed. 2002, 41, 2927; Entwistle, C. D.; Marder, T. B.; Chem. Mater. 2004, 16, 4574.

7. Alcaraz, G.; Euzenat, L.; Mongin, O.; Katan, C.; Ledoux, I.; Zyss, J.; Blanchard-Desce, M.; Vaultier, M.; Chem. Commun. (Cambridge, U.K) 2003, 22, 2766.

8. Reyes, H.; Muñoz, B. M.; Farfan, N.; Santillan, R.; Rojas-Lima, S.; Lacroix, P.; Nakatani, K.; J. Mater. Chem. 2002, 12, 2898.

9. Becker, P.; Adv. Mater. (Wienheim, Ger.) 1998, 10, 979.

10. Keszler, D. A. Em Encyclopedia of Inorganic Chemistry; ed. Chichester: John Wiley \& Sons, 1994, p. 318.
11. Sasaki, T.; Mori, Y.; Kuroda, I.; Nakajima, S.; Yamaguchi, K.; Watanabe, S.; Acta Crystallogr., Sect. C: Cryst. Struct. Commun. 1995, 51, 2222.

12. Procópio, M. B.; Tese de Doutorado, Universidade Federal de Minas Gerais, Brasil, 2009.

13. Greenwood, N. N.; Comprehensive Inorganic Chemistry, $2^{\text {nd }}$ ed., Pergamon Press: Oxford, 1973, Cap. 11.

14. Lide, D. R., Editor-in-Chief; Handbook of Chemistry and Physics, $71^{\text {st }}$ ed., CRC Press: falta cidade, 1990, Cap. 10.

15. Jensen, F.; Introduction to Computational Chemistry, John Wiley \& Sons: New York, 2007.

16. Pople, J. A.; Krishnan, R.; Schlegel, H. B.; Binkley, J. S.; Int. J. Quantum Chem. Symp. S13 1979, 225; Yamaguchi, Y.; Osamura, Y; Schaefer, H. F.; J. Am. Chem. Soc. 1983, 105, 7506; Alter, E. A.; Bartlett, R. J.; J. Chem. Phys. 1989, 3, 1767; Pulay, P.; Mol. Phys. 1969, 17, 197.

17. Helgaker, T.; Jørgensen, P.; Theor. Chim. Acta 1989, 2, 111; Norman, P.; Jonsson, D.; Vahtras, O.; Ågren, H.; Chem. Phys. 1996, 203, 23.

18. Luo, Y.; Ågren, H.; Jørgensen, P.; Mikkelsen, K. V.; Adv. Quantum Chem. 1995, 26, 165.

19. Ruud, K.; Astrand, P. O.; Taylor, P. R.; J. Chem. Phys. 2000, 112, 2668.

20. Torrent-Sucarrat., M.; Solà, M.; Duran, M.; Luis, J. M.; Kirtman, B.; J. Chem. Phys 2003, 118, 711.

21. Frisch, M. J.; Pople, J. A.; Binkley, J. S.; J. Chem. Phys. 1984, 80, 3265.

22. Rassolov, V. A.; Pople, J. A.; Ratner, M. A.; Windus, T. L., J. Chem. Phys. 1998, 109, 1223; Rassolov, V. A.; Ratner, M. A.; Pople, J. A.; Redfern, P. C.; Curtiss, L. A.; J. Comput. Chem. 2001, 22, 976.

23. Schmidt, M. W.; Baldridge K. K.; Boatz, J. A.; Elbert, S. T.; Gordon, M. S.; Jensen, J. H.; Koseki, S.; Matsunaga, N.; Nguyen, K. A.; Su, S. J.; Windus, T. L.; Dupuis, M.; Montgomery, J. A.; Comput. Chem. 1993, 14, 1347; GAMESS: General Atomic and Molecular Eletronic Structura System, http://www.msg.ameslab.gov/GAMESS/GAMESS. html, acessada em Maio 2009.

24. Helgaker et. al.; DALTON, a molecular electronic structure program, Release 2.0, 2005, acessada em Maio 2009.

25. Oudar, J. L.; Chemla, D. S.; J. Chem. Phys. 1977, 66, 2664.

26. Cotton, F. A.; Wilkinson, G.; Advanced Inorganic Chemistry, $5^{\text {th }}$ ed., John Wiley \& Sons: New York, 1999, cap. 6.

27. von Zettler, F.; Hausen, H. D.; H. Hess, H.; Acta Crystallogr., Sect. B: Struct. Sci. 1974, 30, 1876.

28. Bramham, G.; Batsanov, A. S.; Marder, T. B.; Norman, N. C.; Acta Crystallogr., Sect. E: Struct. Rep. Online 2006, 62, o972.

29. Clegg, W.; Marder, T. B.; Scott, A. J.; Wiesauer, C.; Weissensteiner, W.; Acta Crystallogr., Sect. E: Struct. Rep. Online 2001, 57, 063.

30. Hinchliffe, A.; Soscùn, M. H. J.; J. Mol. Struc. 1995, 331, 109.

31. Alparone, A.; Reis, H.; Papadopoulos, M. G.; J. Phys. Chem. A 2006, 110,5909

32. Daniel, C.; Dupuis, M.; Chem. Phys. Lett. 1990, 171, 209.

33. Salek, P.; Helgaker, T.; Vahtras, O.; Ågren, H.; Jonsson, D.; Gauss J.; Mol. Phys 2005, 103439.

34. Soscùn, H.; Castellano, O.; Bermúdez, Y.; Toro, C.; Cubillán, N.; Hinchliffe, A.; Phu, X. N.: Int. J. Quantum Chem. 2005, 106, 1130.

35. Sadlej, A. J.; Theor. Chim. Acta 1991, 79, 123; Sadlej, A. J.; Theor. Chim. Acta 1992, 81, 45; Sadlej, A. J.; Theor. Chim. Acta 1992, 83, 351.

36. Willets, A.; Rice, J. E.; Burland, D. H.; Shelton, D. P.; J. Chem. Phys. 1992, 97, 7590 .

37. Reis, H.; J. Chem. Phys. 2006, 125, 014506.

38. Marder, S. R.; Beratan, D. N.; Cheng, T.; Science (Washington, DC, U.S.) 1991, 252, 103. 